\title{
¿PODEMOS CONFIAR EN LA TECNOLOGÍA? UNA REFLEXIÓN SOBRE LA CRÍTICA SOCIAL EN EL MUNDO TECNOLÓGICO
}

\section{CAN WE TRUST TECHNOLOGY? A PHILOSOPHICAL REFLECTION ON THE MEANING OF TRUST AND SOCIAL CRITICISM IN THE TECHNOLOGICAL WORLD}

\author{
JosÉ A. LÓPEz CEREZO \\ cerezo@uniovi.es \\ Universidad de Oviedo
}

RECIBIDO: 19/06/2020

ACEPTADO: 15/07/2020

Resumen: En este trabajo ensayaremos una reflexión exploratoria sobre el significado de la confianza en la tecnología y el valor del escepticismo crítico en el actual mundo tecnológico. Tras examinar el significado de las nociones de confianza y dependencia, y sobre la base de las aportaciones de diversos autores entre los que destacan U. Beck y P. Sztompka, argumentaremos que la creciente artificialización y despersonalización de nuestro entorno está induciendo un tránsito desde la confianza a la dependencia en los cimientos del tejido social. Se defenderá finalmente el valor del escepticismo personal y la crítica social como respuesta frente a la creciente alienación tecnológica en el mundo actual.

Palabras clave: confianza, dependencia tecnológica, crítica social, artificialización.

\begin{abstract}
In this paper we will try an exploratory reflection on the meaning of trust in technology and the value of critical skepticism in today's technological world. After examining the meaning of the notions of trust and dependence, and based on the contributions of various authors among which U. Beck and P. Sztompka stand out, we will argue that the increasing artificialization and depersonalization of our milieu is inducing a transition from confidence to dependence on the foundations of the social fabric. Finally, the value of personal skepticism and social criticism will be defended in response to the growing technological alienation in today's world.
\end{abstract}

Key words: trust, technological dependence, social criticism, artificialization. 
En la sociedad contemporánea vivimos en un mundo artificial creado por ingenieros. No es algo nuevo. Decía José Ortega y Gasset a mediados del siglo pasado que el ser humano no vive con la técnica sino que vive en la técnica (Ortega, 1951). Nuestras formas de vida y las relaciones con nuestros semejantes dependen estrechamente de artefactos y sistemas tecnológicos.

Se trata no obstante de una relación de dependencia que no simplemente responde a las aspiraciones humanas de cada época, al contrario de lo que pensó Ortega. Las innovaciones tecnológicas necesitan un nicho de oportunidad para salir adelante, pero con su difusión acaban estimulando las necesidades que satisfacen. Como apunta George Basalla (1989: 159ss.), el coche no surgió por una crisis internacional debida a la escasez de caballos. Su inicial uso recreativo terminó configurando el modo de vida actual dependiente del automóvil. El coche no satisface el modo de vida actual sino que lo permite y estimula.

Un autor que a mediados del siglo pasado reflexionó con un lúcido pesimismo sobre este fenómeno es Jacques Ellul (e.g. 1954). El pensador francés veía el desarrollo tecnológico como un proceso autónomo que no nos conduce a mejores formas de vida y al que, sin embargo, no podemos sustraernos. En su visión, la sociedad depende de la tecnología. Autores posteriores como Wiebe Bijker, ya en los años 80, han defendido, contra la visión de Ellul, la maleabilidad y dependencia de ese desarrollo respecto a los agentes humanos (e.g. Bijker et al., 1987). Se trata de la visión más optimista del constructivismo social donde la tecnología depende de la sociedad.

Una visión más equilibrada es la defendida por Thomas Hughes desde los años 80, donde sociedad y tecnología se hallan en una relación de dependencia mutua, intensificándose en un sentido u otro en función de la fase de desarrollo tecnológico (e.g. Hughes, 
1983). Asumiendo ahora, con este autor, una relación de dependencia recíproca donde la tecnología va cobrando autonomía a medida que avanza en su proceso de domesticación, podemos preguntarnos por los motivos para ser optimistas o pesimistas respecto a su evolución futura.

El propósito de este ensayo no es tanto responder a la cuestión anterior como explorar un modo particular de plantearla y examinar las consecuencias que se derivan respecto al significado de vivir en una sociedad tecnológica. En efecto, para fundamentar mejor nuestra inclinación hacia el optimismo o el pesimismo, la pregunta que deberíamos formular es si tenemos motivos para confiar en la tecnología, y, en cualquier caso, ¿qué significa, si es que significa algo, “confiar en la tecnología”?

\section{La noción de confianza}

Comencemos centrándonos ahora en el concepto mismo de confianza para explorar más adelante la "confianza tecnológica".

En sociología y ciencias políticas, la confianza es descrita con frecuencia como un constructo social necesario para la vida en común (e.g. Weber y Carter, 2003: 4-5). La confianza hace posible la gobernanza a través de la producción de capital social, o, como diría Durkheim (1893/1987) desde la tradición sociológica, densidad moral, es decir, intensidad de comunicaciones e intercambios entre individuos. Pero es algo que va más allá de la interacción entre individuos. Nos movemos en un entorno de expertos, sistemas y artefactos que suponemos saben lo que hacen y funcionan como deberían. Es precisamente la ubicuidad de la confianza lo que la hace invisible (Stern, 2017: 274).

La noción de confianza es no obstante una noción compleja. En tanto que virtud intelectual y virtud moral, incluye capacidades 
cognitivas y afectivas imposibles de describir mediante un algoritmo. Como ya señalaba Aristósteles en la Retórica al hablar de la persuasión, la confianza que depositemos en un interlocutor depende no solo de la sabiduría práctica (phrónesis) que demuestre sino también del carácter virtuoso (areté) y de la buena voluntad o benevolencia (eunoia) que manifieste.

En tanto que actitud orientada al futuro, hay ciertos aspectos que es necesario resaltar en la noción de confianza: la expectativa, la vulnerabilidad y el riesgo (Hupcey et al., 2009: 290; McCraw, 2015: 416-18). La confianza puede entenderse en términos de lo que esperamos respecto a nuestros semejantes y el mundo que nos rodea, $y$, en este sentido, involucra la expectativa de la realización de alguna acción o estado de cosas (el objeto diana de la confianza). Pero el agente que confía, en tanto que dependiente de otro sujeto o institución para la realización de lo esperado, se sitúa en una posición vulnerable respecto al fracaso o incumplimiento. La confianza también implica riesgo puesto que requiere considerar cursos de acción y la elección entre alternativas (confiar/desconfiar), colocándonos entonces ante una posible expectativa defraudada o bien ante una posible pérdida de oportunidad.

En su contribución pionera Trust and Power, Niklas Luhmann entiende la confianza como un mecanismo de reducción de la complejidad, subrayando la relación entre la noción de confianza y las nociones de riesgo y expectativa. Para este autor, la confianza constituye una apuesta arriesgada de anticipación del futuro, consiste en actuar como si cierto futuro fuese a ser el caso. En sus palabras:

El problema de la confianza por tanto consiste en el hecho de que el futuro contiene muchas más posibilidades de las que pueden realizarse en el presente y, por consiguiente, ser transferidas al pasado.... El futuro coloca una carga excesiva sobre la capacidad de representación del 
hombre. Debe por tanto podarse el futuro para acomodar el presente - i.e. reducir la complejidad" (1979: 13).

La atribución de confianza "fija” así el comportamiento futuro de otros actantes ${ }^{1}$ como presupuesto para decidir el comportamiento propio en el presente, reduciendo la incertidumbre y haciendo posible la acción social.

Pero ¿en qué sentido confiamos cuando decimos confiar en, por ejemplo, otra persona? Desde un punto de vista estructural, podemos entender la confianza como una relación cuatriádica donde un actor (trustor) confía en otro actor (trustee) respecto a cierta acción o estado de cosas, dado cierto contexto (e.g. Zagzebski, 1966; Blackburn, 1998; McCrew, 2015).

Dado cierto contexto X, A confía en B respecto a C

Con respecto a la propia relación, y de acuerdo con la literatura especializada (e.g. Renn, 2008: 222), a pesar de los debates puntuales en estudios empíricos, hay tres dimensiones principales en la confianza que afectan al trustee, y por tanto tres modos de entenderla o de ponderar sus factores: la competencia percibida (el grado de conocimiento o pericia, incluyendo la eficiencia), la independencia e integridad percibida (falta de sesgo, objetividad, consistencia, sinceridad), y la empatía percibida (similitud de valores o cosmovisiones, benevolencia). Son dimensiones aproximadamente coincidentes con los factores que, de acuerdo con la gran obra clásica sobre la persuasión (Retórica de Aristóteles), hacen a alguien digno de crédito (Mayer et al., 1995:717). ${ }^{2}$

${ }^{1}$ Actores humanos o no humanos, en el sentido de la teoría del actor-red. E.g. Callon (1986).

2 De hecho, la credibilidad suele entenderse como una manifestación de la confianza en el ámbito de la comunicación (e.g. Renn, 2008: 223; Walaski, 2011: 
La diferenciación depende en última instancia de la clase de expectativas implicadas por el ejercicio de la confianza. En la terminología sociológica, en el primer caso se trata de expectativas instrumentales relativas a aspectos técnicos o epistémicos, en el segundo de expectativas axiológicas relacionadas con valores morales, y en el tercero de expectativas fiduciarias relativas a la anteposición del interés ajeno por delante del propio (Sztompka, 2000: 53 ss.). Hay que tener en cuenta que la atribución de credibilidad o el ejercicio de la confianza suele combinar diferentes modalidades (competencia, independencia, empatía) y además puede hacerlo con distintas intensidades en un continuo.

Decíamos antes, al hablar de la vulnerabilidad implicada en la confianza, que el agente que confía, en tanto que dependiente de otro sujeto o institución (o quizá sistema técnico) para la realización de lo esperado, se sitúa en una posición vulnerable respecto al posible incumplimiento. Los conceptos de confianza (trust) y dependencia (dependence/reliance) presentan relevantes paralelismos e importantes diferencias. Tienen la misma estructura relacional cuatriádica, implican vulnerabilidad y riesgo, y coinciden también en no presuponer (a diferencia de un contrato o una promesa) una autoridad u obligación que pueda requerir a $\mathrm{B}$ el cumplimiento de $\mathrm{C}$, de modo que $\mathrm{B}$ adquiera una firme responsabilidad legal o moral como depositario de la confianza o agente de la dependencia (Darwall, 2017).

El concepto de confianza es no obstante más restringido que el de dependencia: constituye una forma voluntaria de dependencia (Rousseau et al., 1998: 394-395). La confianza implica dependencia pero no a la inversa, pues podemos depender de otras personas aunque desconfiemos de ellas (Zagzebski, 1996: 160). La dependencia, además, y a diferencia de la confianza, no requiere la existencia de una expectativa respecto al cumplimiento de $\mathrm{C}$ ni

20ss.)

Argumentos de Razón Técnica, n 23, 2020, pp. 13-36 
presupone la buena voluntad (o capacidad) del depositario de la misma y por tanto no puede ser defraudada o traicionada (O'Neil, 2017). Aunque ambos conceptos implican vulnerabilidad, en la confianza el riesgo deber ser reconocido y asumido, algo que no ocurre en la dependencia, pues en esta, al contrario que en la confianza, no hay capacidad de elección (depender/no depender).

\section{La confianza tecnológica}

Un informe de referencia de la OCDE, publicado de 2017, distingue varias formas de confianza: confianza en otras personas (confianza interpersonal), confianza en organizaciones e instituciones (confianza institucional), confianza en intangibles como marcas (brand trust) y confianza en sistemas, objetos y tecnologías (confianza tecnológica) (OCDE, 2017: 17).

Dejando de lado la confianza en intangibles, que podría fácilmente reducirse a confianza institucional o confianza tecnológica (dependiendo del objeto de la confianza), hay en la clasificación anterior dos cuestiones importantes para nosotros. La primera es si podemos hablar de confianza institucional como una variedad estricta de la confianza, o si se trata más bien un tipo de confianza derivada, es decir, que tiene como diana a las personas mediante las cuales se visibilizan esas instituciones (representantes políticos, directores generales, empresarios). La segunda cuestión, análogamente, es si podemos hablar de confianza tecnológica como una variedad estricta de la confianza, o si es más bien un tipo de confianza derivada de segundo grado, es decir, que tiene como diana a las personas mediante las cuales se visibilizan las instituciones de las que, a su vez, depende el diseño, desarrollo y comercialización de esas tecnologías (ingenieros, empresarios). 
Un lugar bastante común en la literatura (e.g. Warren 2018: 83 y 88) es que la forma básica de la confianza es la confianza social o interpersonal, de modo que la confianza institucional sería una forma de confianza interpersonal respecto a un agente, anónimo o no, de quien depende la institución. ¿Qué ocurriría en este caso con la confianza tecnológica? ¿Puede la dependencia voluntaria respecto a sistemas, objetos y tecnologías entenderse como una forma de confianza?

En principio, tendemos a pensar que una máquina es fiable o no fiable (reliable/not reliable), más que confiable o no confiable (trustable/not trustable), y respecto a su funcionamiento o desempeño regular nuestra disposición es hacia la confidencia (confidence), más bien que la mera confianza. ${ }^{3}$ ¿Pero por qué decimos sin embargo confiar en un reloj suizo o un coche japonés, y no lo hacemos de un reloj chino o un coche italiano? ¿Se trata de un uso metafórico del término o de un tropo retórico? La respuesta obvia es porque sabemos que el reloj suizo no se va a parar a media mañana o el coche japonés dejarnos tirados en la carretera. ¿Pero quién o qué genera las expectativas (y crea la vulnerabilidad) que son la base de esa confianza? ¿Es experiencia (información) relativa a las características de la máquina o de máquinas de esa clase, o bien relativa al prestigio de la empresa fabricante o de ese

${ }^{3}$ No es sencillo diferenciar en español entre los términos ingleses trust y confidence, que suelen traducirse igualmente como "confianza" y usarse como sinónimos. Parece no obstante apropiado utilizar el término "confidencia” para confidence. Una de las acepciones del diccionario de la RAE para el término castellano "confidencia" es "confianza estrecha e íntima”, es decir, la seguridad firme que se tiene en alguien o algo, como que la luz se encenderá por la mañana al accionar el interruptor (Siegrist et al., 2007: 268). Lo importante aquí es que en la confidencia no se supone que uno deba reunir evidencia para asumir la actitud ni colocarse tampoco voluntariamente en una situación de dependencia, como sí se presupone con la confianza (Giddens, 1997: 31; Hupcey et al. 2001: 290). 
tipo de empresas (confianza derivada), o más bien sobre los ingenieros y gestores vinculados habitualmente a la imagen pública de la empresa (confianza derivada de segundo grado)?

Recientemente, en un corto periodo temporal, se han estrellado dos aviones Boeing del mismo modelo: 737 MAX 8 (octubre de 2018 y marzo de 2019). Las autoridades aeronáuticas de muchos países suspendieron los vuelos de dicho modelo, incluidos EE.UU. y Europa, y los mantienen suspendidos un año después. La sospecha principal recae sobre un sistema automático de nivelación del vuelo en el despegue (MCAS), que en ciertas circunstancias puede nivelar en exceso el morro del avión si detecta un ángulo con una inclinación muy elevada, y que los pilotos parecen no controlar adecuadamente. Como consecuencia, muchas líneas aéreas han cancelado sus pedidos del ese modelo, causando un grave problema en la bolsa para la empresa. La FAA norteamericana incluso ha vertido sospechas sobre Boeing respecto a haber ocultado información acerca de dicho sistema o actuado negligentemente en el proceso de certificación (donde se subcontrata a la propia Boeing) - necesario para que un modelo de avión reciba la autorización para vuelos comerciales.

¿Qué estamos diciendo cuando decimos que se ha perdido la confianza en el 737 MAX (y, como consecuencia, se ha deteriorado la confianza en Boeing)? Desde luego, si todo el proceso acaba judicializándose, podría acabar con una multa para la empresa o, si se encuentran indicios de conducta criminal o negligente, con alguna persona encarcelada. El primer caso expresaría un resultado que afecta a la confianza institucional, y en el segundo a la confianza personal. En casos como este, no es infrecuente que la propia empresa presente un chivo expiatorio sobre el que recaiga la responsabilidad, de modo que se limiten los daños para la institución. El culpable es presentado entonces como una manzana podrida que no fue adecuadamente detectada por los mecanismos 
de control, desviando así el foco de las estructurales institucionales que favorecen o hacen posible la aparición de tales casos.

¿Qué se ha perdido, en cualquier caso, con el accidente de esos dos aviones (aparte de las máquinas y las vidas implicadas)? La erosión de la confianza podría decirse que afecta a los tres niveles señalados: a nuestras expectativas sobre el funcionamiento de la máquina, sobre las empresas e instituciones implicadas, y sobre la capacidad de las personas (ingenieros y reguladores) concernidas. En un sentido contrario, se nos pide hoy que confiemos en el coche autónomo, pues se trata de un futuro irrenunciable. ¿Por qué debemos confiar en él? Son en principio posibles tres líneas de respuesta: por la calidad de los prototipos y su comportamiento en las pruebas, por el prestigio de las empresas que los fabrican y las instituciones que los regulan (incluyendo los mecanismos de compensación en caso de daños), e implícitamente por la destreza de los ingenieros que los diseñan. Plausiblemente, cuando decimos confiar o desconfiar en el coche autónomo, estamos combinando estas líneas de respuesta, es decir, estamos conectando la confianza tecnológica con la confianza institucional y con la confianza interpersonal (Bellow, 2017: 128-132). ¿Se produce realmente algún tipo de transitividad en esta recursión entre niveles?, ¿cuál es el papel de la dependencia?, ¿dónde nos lleva esta conexión?

\section{La sociedad tecnológica}

Decía Ortega (1939) que la técnica constituye la proyección intelectual y realización material de los proyectos de vida. En principio, parece que podemos confiar o desconfiar, y, en algunos casos, optar por la dependencia o no dependencia respecto a la tecnología. ¿Qué tipos de proyectos de vida pueden resultar de esas combinaciones? 
Confiar y aceptar encantados la dependencia definiría la actitud tecno-optimista de autores como Alvin Toeffler, José Manuel Rodríguez Delgado o los numerosos buhoneros del transhumanismo o el antropocenismo. Combinar la desconfianza y el reconocimiento de la dependencia es la postura característica del tecnopesimismo de Jacques Ellul o Langdon Winner. Rechazar la dependencia sin abrazar la desconfianza (no llegar a confiar no implica desconfiar, es solo no confiar $)^{4}$ podría ser la actitud característica de los Amish norteamericanos, cuyos motivos religiosos les llevan a prescindir de numerosos avances tecnológicos. Y, por último, combatir la dependencia sobre la base de la desconfianza es la actitud propia de personajes como los que protagonizan las novelas Into de Wild o Zen and the Art of Motorcycle Maintenance: jóvenes idealistas como Alex Supertramp que rechazan el adocenamiento tecnológico de la vida burguesa y buscan el reencuentro con la naturaleza.

Como muestran esas mismas noveles, es imposible la independencia completa respecto a la tecnología en el mundo artificial donde vivimos. ¿Qué papel juega en el mismo la confianza? ¿Hay un espacio para la confianza tecnológica?

Hay ciertos los rasgos de la sociedad contemporánea que, desde luego, confieren una particular relevancia a la confianza, y particularmente a la confianza social. Para examinarlos podemos apoyarnos en el trabajo de Ulrick Beck y Piotr Sztompka. De acuerdo con Beck (1986) y su visión del mundo contemporáneo como una "sociedad del riesgo", hemos pasado de una sociedad basada en el destino a otra modelada por la acción humana intencional, con un poder creciente de modificación de la vida

\footnotetext{
4 “Confiar” y "desconfiar" son términos contrarios, no contradictorios. No pueden ser ambos verdaderos pero sí ambos falsos. Sin embargo, "ser dependiente" y "ser independiente" expresan juicios contradictorios: si uno es verdadero, el otro es falso, y viceversa.
} 
social y del entorno. ${ }^{5}$ El desarrollo tecnológico e industrial ha producido nuevos tipos de amenaza y ha llevado el riesgo al centro de la vida social $\mathrm{y}$ personal, generando incertidumbre $\mathrm{y}$ vulnerabilidad.

Forma parte de un fenómeno más amplio de artificialización creciente del entorno e incluso de las relaciones interpersonales. La administración pública y el mercado, mediante artefactos materiales o burocráticos, tienen cada vez mayor presencia al posibilitar, condicionar o regular los diversos ámbitos de la conducta humana. Un creciente rango de bienes y servicios, que tradicionalmente era gratuitos y cuya provisión dependía de familiares, amigos o la simple naturaleza, ahora depende de administraciones o empresas: el cuidado de los mayores, el consejo y respaldo emocional, el ocio, los pequeños placeres cotidianos, el ejercicio y deporte, y hasta el agua y aire saludables.

Debemos también tener en cuenta, según Stzompka (2000), el alto grado de diferenciación funcional, interdependencia y globalización que hemos alcanzado en nuestra "sociedad del riesgo”. La complejidad creciente de las instituciones y los sistemas tecnológicos, su interdependencia y el alcance global de su operación, ha hecho que grandes segmentos del mundo social sean opacos para sus miembros. Y, a su vez, la opacidad de organizaciones e instituciones ha producido el creciente anonimato y despersonalización de aquellos de los que depende nuestro

${ }^{5}$ Una de las características centrales de la llamada "sociedad del riesgo" es que las amenazas actuales ya no se conceptualizan como peligros, es decir, como daños inevitables. Prácticamente todos los males que hoy nos amenazan son entendidos como riesgos, es decir, como daños que resultan de la acción o de la omisión de la acción de algún ser humano. En el pasado, y quizá todavía en algunas culturas fuertemente ancladas en la tradición o en los márgenes remotos del globo, los males se atribuían al destino, a la naturaleza o a alguna voluntad sobrenatural. En la sociedad del riesgo son motivo de atribución de responsabilidad a algún agente social. 
bienestar. Desconocemos a los agentes que están detrás del sistema financiero, los científicos que elaboran nuevos principios activos en medicamentos o los ingenieros responsables del diseño de los aviones: la confianza social es una apuesta acerca de las acciones contingentes de opacos agentes institucionales, un mecanismo para manejar un futuro incierto e incontrolable (Earle y Cvetkovich, 1995: 9, 21ss.).

¿Qué función juega en este contexto la confianza tecnológica, si es que podemos hablar de tal cosa? Las instituciones tienden a la opacidad y las cosas también, no solo para los usuarios sino incluso para aquellos cuya profesión implica la interacción regular con esos objetos. Pensemos en un objeto tecnológico como un coche. En el pasado si la carrocería se abollaba, el chapista lo arreglaba. Ahora cambian la moldura completa. En el pasado, si se estropeaba un componente del motor, el mecánico arreglaba la pieza defectuosa. Ahora cambia el bloque entero al que pertenece esa pieza. Tal opacidad, en tanto que pérdida de transparencia y limitación de la participación, constituye una cualidad alienante de la tecnología, en términos de Miguel Ángel Quintanilla (2017, 2018). Realizar hoy una gestión en una administración púbica también es acceder a una maquinaria burocrática anónima en la que nuestro margen de acción está muy constreñido.

La confianza parece requerir algún tipo de referente personal, derivado o no. Desde un planteamiento tradicional, como hemos visto, la voluntariedad de la dependencia (confianza) respecto a la tecnología podía ser entendida como una modalidad derivada de la confianza institucional (que, a su vez, se fundamentaba en última instancia en la confianza interpersonal). ${ }^{6}$ En sentido estricto,

${ }^{6} \mathrm{Si}$ bien la resiliencia de las instituciones, es decir, el modo en que sus rasgos estructurales tienden a producir un funcionamiento adecuado a pesar de las limitaciones de los individuos que forman parte de ellas, puede generar confianza institucional a pesar de la desconfianza que puedan suscitar los individuos 
dependíamos de la tecnología (fiable o no) y tendíamos a confiar en las instituciones que la producían y regulaban. Con la artificialización creciente y despersonalización de la sociedad del riesgo, las tornas tienden a invertirse y la confianza institucional se presenta como una modalidad derivada de la dependencia tecnológica. Servicios públicos tan comunes como el cuidado de la salud, el pago de los impuestos o el desplazamiento en un avión, tienden a burocratizarse, despersonalizarse y adaptarse a un procedimiento mecánico que minimiza riesgos y optimiza beneficios.

Es una línea de razonamiento que conduce a una sospecha: debido a la opacidad y despersonalización creciente de las instituciones, así como mediante la interposición creciente de sistemas técnicos y procedimiento mecánicos en las relaciones interpersonales, estamos en general transformando trust en reliance, la confianza tiende a transformarse en dependencia, y en particular la confianza institucional tiende a transformarse en una variedad de la dependencia tecnológica. Los ejemplos no son escasos, particularmente si prestamos atención a la mecanización y artificialización de la relación entre la prestación de servicios o provisión de bienes y los usuarios de esos servicios o consumidores de esos bienes. Todos hemos compartido la frustración del servicio automatizado de información telefónica, la tortura de la actualización automática del software o el sufrimiento de la gestión burocrática de un gran número de trámites.

La cadena de la confianza "personas $\rightarrow$ instituciones $\rightarrow$ máquinas", donde la atribución de la cualidad depende del

asociados a dichas instituciones. En este sentido se manifiesta un reciente estudio sobre valores y actitudes de los europeos acerca de la esfera pública (Fundación BBVA, 2019). Para evitar la corrupción, si desconfiamos de los individuos, puede funcionar bien un sistema robusto de rendición de cuentas - siempre que presupongamos en ellos un nivel mínimo de competencia (Sandman, 1993; Löfstedt, 2009) 
elemento anterior, se invierte y se transforma en una relación de dependencia "máquinas $\rightarrow$ instituciones $\rightarrow$ personas". La dependencia, en tanto que subespecie involuntaria de la confianza, asume el papel principal definitorio de nuestras relaciones con las cosas, los organismos y las personas. En lugar de confiar en los artefactos porque confiamos en las instituciones que los fabrican (por contar con ingenieros competentes), estamos a merced de personas anónimas por el modo en que los artefactos nos hacen dependientes de opacos organismos. De este modo, la creciente despersonalización y artificialización de nuestro entorno parece estar induciendo un tránsito desde la confianza a la dependencia en los cimientos del tejido social.

No hay ninguna ley o conspiración responsable. Es simplemente un nuevo estado de cosas que tiende a consolidarse: en el pasado quizá la tecnología respondía en cierta medida a las aspiraciones sociales, en el presente la sociedad tiende a responder y adaptarse a las posibilidades que ofrece la Tecnología, en el mismo sentido en que Ellul decía ya en 1954 que el fenómeno técnico (la "Technique" con mayúscula) tendía a dominar y engullir toda otra forma de actividad humana.

\section{La sociedad postconfianza}

Un término que ha adquiero cierta notoriedad en nuestros días es el de "sociedad de la postconfianza" (Löfstedt, 2009: xix), como fase del desarrollo de nuestro sociedad tecnológica postindustrial. La opinión pública, aun teniendo en cuenta el efecto de amplificación del riesgo regularmente generado en los medios (Kasperson, 1992), se muestra cada vez más recelosa con respecto a los mensajes de la industria y las tradicionales autoridades reguladoras respecto a la seguridad de los productos y sistemas 
tecnológicos. Es una consecuencia de las crisis alimentarias, sanitarias e industriales de las últimas décadas, ${ }^{7}$ sobre el trasfondo de las protestas originarias de los años 60 contra la energía nuclear $\mathrm{y}$ el deterioro de la naturaleza, y han terminado produciendo una importante erosión de la confianza en los poderes públicos, como por ejemplo pone de manifiesto el World Values Survey y otros estudios demoscópicos (OCDE, 2017: 19 ss.).

Pero la sociedad postconfianza no es una sociedad de la desconfianza. Desde el punto de vista tradicional, la confianza genera una gestión eficiente a través de la producción de capital social (e.g. OCDE, 2017). La confianza es sin embargo solo una forma de fundamentar una gestión eficiente. Cuando la confianza en las personas u organismos falla, seguimos estando dispuestos a depender de ellos siempre que contemos con estructuras institucionales apropiadas, como leyes que se cumplan, independencia judicial, transparencia en la gestión, rendición de cuentas de los poderes públicos y oportunidades de participación. Son estructuras diseñadas para eliminar la necesidad de confianza o al menos reducir los costes en caso de que esa confianza se debilite (Löstedt, 2009: xi).

¿Qué ocurre sin embargo cuando esas leyes son insuficientes, las organizaciones son opacas y los gestores anónimos, o la participación es meramente instrumental? Lo que sucede es que la confianza deja paso a la dependencia, que la sociedad postconfianza devine una sociedad de la dependencia. Lo que constituía una magnífica oportunidad para promover el

\footnotetext{
${ }^{7}$ A modo de ejemplo: la sangre contaminada con SIDA en Francia a mediados de los 90, la gripe aviar de finales de los 90, la crisis de las vacas locas en la Europa de los años 90, el antiinflamatorio Vioxx en EE.UU. a principios de 2000 y otros muchos fármacos (Lipobay, Trasylol, Jasmin, etc.), la leche materna contaminada en China en 2008 o, más recientemente, los automóviles trucados fabricados por Volkswagen o el tráfico de datos de Cambridge Analytics
} 
protagonismo ciudadano y la profundización democrática se convierte en un mecanismo de alienación.

En un proceso sin evidencias pero con indicios crecientes: el tránsito desde una sociedad de la confianza hacia una sociedad de la dependencia. Los riesgos voluntariamente asumidos, sobre la base de expectativas, se transforman nuevamente en peligros aparentemente inevitables que dependen de fuerzas más allá de nuestro control. Regresamos al pasado: convertimos la vida social en una megamáquina, en el sentido de Mumford (1970), ${ }^{8}$ y le conferimos autonomía. Como en la ficción distópica de James G. Ballard en Rascacielos (1975), el espacio que nos rodea y las cosas que contiene, artefactos materiales y sociales, condiciona nuestro comportamiento y va modelando nuestra conciencia, generando hoy dependencia y alienación. La opción ya no es confiar o no en las instituciones, o en los sistemas tecnológicos, sino tratar de minimizar nuestro grado de dependencia y vulnerabilidad. No solo las tecnologías necesitan hacerse "entrañables" (en el sentido de Quintanilla, 2018) sino también las instituciones que imitan tecnologías indeseables. ${ }^{9}$

${ }^{8}$ Es decir, una organización social rígida y jerárquica que produce grandes beneficios materiales a costa de la deshumanización y las limitaciones personales (Mumford, 1970).

9 En su decálogo de tecnologías entrañables, M.A. Quintanilla (2017: cap. 1) incluye las siguientes virtudes: que sean abiertas (sin restricciones de uso, copia, modificación o distribución), polivalentes (que permitan usos alternativos), dóciles (que su funcionamiento, control y parada dependa de un ser humano), limitadas (con consecuencias sociales y para el medio natural predecibles), reversibles (siendo posible restaurar el medio natural o social en el que se implante un sistema técnico), recuperables (susceptibles de recuperación y mantenimiento activo, sin obsolescencia programada), comprensibles (abiertas al entendimiento del sistema por ciudadanos con una formación cultural básica), diseño participativo (con un desarrollo abierto a la participación ciudadana), sostenibles (que hagan posible el ahorro y reciclado), y socialmente responsables (que tengan en cuenta la desigualdad social y la situación de los más desfavorecidos). 


\section{Vivir en la tecnología}

Las nuevas realidades sociales definidas por una vertiginosa transformación tecnológica de nuestras condiciones de vida, más la obsesión por la seguridad y aversión al riesgo generadas por la evolución reciente del escenario geopolítico y sociosanitario mundial, han creado nuevas tensiones a las que debe hacer frente nuestra sociedad postconfianza.

Primero la tensión entre independencia y condicionamiento, entre la búsqueda de espacios para el desarrollo en libertad del propio proyecto de vida, en términos de Ortega, y la necesidad constante de adaptarnos a los nuevos formatos de productividad e interacción social generados por la irrupción constante de nuevos dispositivos y aparatos, así como reglamentos. Quizá de un modo un tanto exagerado pero en este mismo sentido, el Papa declaraba en junio de 2015 que las nuevas tecnologías "dañan el alma” ( $A B C$, 06-06-2015). Segundo la tensión entre autonomía y seguridad, que todos experimentamos con enfado o resignación al tomar un avión o al comprobar el gran avance de organismos opacos (gobiernos, empresas) en el control de la vida privada de los ciudadanos. Tercero la tensión entre libertad individual y responsabilidad social, donde debemos adaptar nuestra conducta y nuestra conciencia a las normas y dispositivos de uniformización social por mor de la salud pública. La propia distinción entre público/privado es dependiente de la disponibilidad de nuevas tecnologías (y "oportunidades de negocio") que transformen aspectos y actividades humanas hasta ahora privadas en objetos de consumo o servicios sujetos a las leyes del mercado y sometidos a organismos reguladores. La amistad, la sinceridad, la salud, los hijos y hasta el aire puro en algunas ciudades son ahora productos de consumo o servicios técnicos que ofrecen las empresas y regulan los poderes públicos. 
Una respuesta a esas tensiones la encontramos en la literatura filosófica: la ética del no poder de Jacques Ellul, la apuesta por la sociedad convivencial frente al fascismo tecnoburocrático de Ivan Illich, la rebelión racional frente a la tiranía de las cosas en Herbert Marcuse, etc. Es decir, asumir un protagonismo civil que, sobre la base del reconocimiento de las nuevas realidades, adopte una actitud crítica, reflexiva y bien informada. Ciertamente, nosotros no podemos escapar del mundo artificial creado por las nuevas tecnologías, pero quizá sí aprender a convivir con el moderno Prometeo, haciéndonos eco de la célebre novela gótica de Mary Shelley.

El reto es entonces cultivar la conciencia crítica y el distanciamiento que haga posible la reflexión pausada (Marcos, 2018). No es algo nuevo resaltar el valor de la crítica para la formación de opiniones y la toma de decisiones. En nuestro tiempo, podemos encontrar las raíces de esta idea en el pensamiento ilustrado del siglo XVIII (Phillips, 2015: 25), por ejemplo en los escritos de Thomas Jefferson y otros pioneros de las revoluciones americana y francesa; más tarde, expresada con rotundidad por John Stuart Mill en Sobre la libertad: "Quien sólo conozca un aspecto de la cuestión no conoce gran cosa de ella (...) si no existieran impugnadores de las verdades fundamentales sería indispensable imaginarlos y proveerlos con los argumentos más fuertes que pudiera inventar el más hábil abogado del diablo” (Mill, 1859: 100-101). De hecho, el término “crítica” procede del griego kritikos, que hace referencia a una persona con buen juicio.

Desde un punto de vista demoscópico, se trata de las actitudes críticas y reflexivas que pueden hallarse en los "escépticos leales" de Martin Bauer y colaboradores (2012), los distrustful engagers de la encuesta británica PAS de 2014 (Ipsos MORI, 2014) o la población "mucho-mucho" de la Encuesta Iberoamericana de 2007 (FECYT-RICYT-OEI, 2009). Son ciudadanos muy interesados en 
la ciencia y que se sienten informados al respecto, con opiniones cualificadas y diferenciadas respecto a distintos ámbitos y aplicaciones de la ciencia y la tecnología. Piensan que la cienciatecnología tiene un gran potencial de beneficios para la sociedad aunque son conscientes y cautelosos respecto a los riesgos y condicionantes que acompañan a su desarrollo. Son los denominados también “ciudadanos CTS” (López Cerezo y Laspra, 2018).

En un mundo en continua y acelerada transformación por efecto de la ciencia y la tecnología, con una creciente artificialización y despersonalización de nuestras condiciones de vida, y con una creciente dependencia de los resortes del desarrollo respecto organizaciones e intereses opacos, una cierta dosis de escepticismo y actitudes críticas en una ciudadanía familiarizada con la ciencia y la tecnología es hoy fundamental para generar espacios de protagonismo ciudadano y disminuir nuestra vulnerabilidad frente a la megamáquina contemporánea. "Seamos realistas, pidamos lo imposible”, decía un conocido slogan de mayo del 68 francés. La crítica y la libertad intelectual no son un problema sino un recurso muy valioso en el actual mundo tecnológico.

\section{Referencias}

Aristóteles, On Rethoric: A Theory of Civic Discourse, G.A. Kennedy (traducc.), $2^{\mathrm{a}}$ ed., Nueva York-Oxford, Oxford University Press, 2007.

Ballard, J.G., Rascacielos, Madrid, Alianza, 2018 (ed. orig. 1975). Basalla, G., The Evolution of Technology, Cambridge, Cambridge University Press, 1989. 
Bauer, M.W., R. Shukla y N. Allum (eds.), The Culture of Science: How the Public Relates to Science Across the Globe, Nueva York, Routledge, 2012.

Beck, U., Risk Society: Towards a New Modernity, Londres, Sage, 1992 (ed. orig. 1986).

Bellow, B., "Who Earns the Trust in Citizens and Why: Experiences from the Private Sector", en: OECD, Trust and Public Policy: How Better Governance Can Help Rebuild Public Trust, Paris, OCDE Publishing, 2017.

Bijker, Wiebe E., Thomas P. Hughes y Trevor Pinch (eds.), The Social Construction of Technological Systems, Cambridge (Mass.), MIT Press, 1989 (ed. orig. 1987).

Blackburn, S., "Trust, Cooperation, and Human Psychology”, en: V. Braithwaite y M. Levi (eds.), Trust and Governance, Nueva York, Russell Sage Foundation, 1998.

Callon, M. (1986), "The Sociology of an Actor-Network: The Case of the Electric Vehicle", en: M. Callon, J. Law y A. Rip (eds.), Mapping the Dynamics of Science and Technology: Sociology of Science in the Real World, Basingstoke, Macmillan, 1986.

Darwall, S., "Trust as a Second-Personal Attitude (of the Heart)", en: P. Faulkner y T. Simpson (eds.), The Philosophy of Trust, Oxford, Oxford University Press, 2017.

Durkheim, E., La división del trabajo social, Madrid, Akal, 1987 (ed. orig. 1893).

Earle, T.C. y G. Cvetkovich, Social Trust: Towards a Cosmopolitan Society, Westport, Praeger, 1995.

Ellul, J., The Technological Society, Nueva York, Vintage, 1964 (ed. orig. 1954).

FECYT-OEI-RICYT, Cultura científica en Iberoamérica. Encuesta en grandes núcleos urbanos, Madrid, FECYT, 2009. En: https://icono.fecyt.es/sites/default/files/filepublicaciones/culturacie ntificaeniberoamerica.pdf (acceso: 19/06/2020). 
Fundación BBVA, Estudio internacional de valores, Madrid, Fundación BBVA, 2019. En: https://www.fbbva.es/wpcontent/uploads/2019/09/Presentacion_Estudio_Valores_2019.pdf (acceso: 19/06/2020).

Giddens, A., The Consequences of Modernity, Stanford, Polity Press, 1997.

Hughes, T.P., Networks of Power: Electrification in Western Society, 1880-1930, Baltimore, Johns Hopkins University Press, 1983.

Hupcey, J.E., J. Penrod, J.M. Morse \& C. Mitcham, "An Exploration and Advancement of the Concept of Trust", Journal of Advanced Nursing, 3/2 (2001): 282-293.

Illich, I., La convivencialidad, Barcelona, Barral, 1974 (ed. orig. 1973).

Ipsos MORI, Public Attitudes to Science 2014, 2014. En: https://www.ipsos.com/sites/default/files/migrations/enuk/files/Assets/Docs/Polls/pas-2014-main-report.pdf

(acceso: 19/06/2020).

Kasperson, R., "The Social Amplification of Risk: Progress in Developing an Integrative Framework of Risk", en: S. Krimsky y D. Golding (eds.), Social Theories of Risk, Westport, Praeger, 1992.

Krakauer, J., Into the Wild, Nueva York, Random House, 1996.

Löfstedt, R.E., Risk Management in Post-Trust Societies, Londres, Earthscan 2009.

López Cerezo, J.A., La confianza en la sociedad del riesgo, Barcelona, Sello Editorial, 2018.

López Cerezo, J.A. y B. Laspra, "The Culture of Risk: STS Citizens Facing the Challenge of Engagement”, en: B. Laspra y J.A. López Cerezo (eds.), Spanish Philosophy of Technology, Dordrecht, Springer, 2018.

Luhmann, N., Trust and Power, Nueva York, Riley, 1979. 
Marcos, A., "Silencio tecnológico", Scio: Revista de Filosofía, 15 (2008): 157-176.

Marcuse, H., One-Dimensional Man: Studies in the Ideology of Advanced Industrial Society, Londres-Nueva York, Routledge, 1964.

Mayer, R.C., J.H. Davis y F.D. Schoorman, “An Integrative Model of Organizational Trust”, Academy of Management Review, 20/3 (1995): 709-734.

McCraw, B.W., "The Nature of Epistemic Trust”, Social Epistemology, 29/4 (2015): 413-430.

Mill, J. S., Sobre la libertad, Madrid, Alianza, 1981 (ed. orig. 1859).

Mumford, L., The Myth of the Machine: The Pentagon of Power, Nueva York, Harcourt, 1970.

OCDE, Trust and Public Policy: How Better Governance Can Help Rebuild Public Trust, París, OECD Publishing, 2017.

O’Neil, C., "Betraying Trust”, en: P. Faulkner y T. Simpson (eds.), The Philosophy of Trust, Oxford, Oxford University Press, 2017.

Ortega y Gasset, J., Meditación de la Técnica, Madrid, Revista de Occidente, 1939.

Ortega y Gasset, J., "El mito del hombre allende la técnica", conferencia en Darmstadt 1951, en: J.A. López Cerezo y J.L. Luján López (eds.), Filosofía de la tecnología, número monográfico de Teorema XVII/3, 1998.

Phillips, A., Journalism in Context: Practice and Theory for the Digital Age, Londres-Nueva York, Routledge, 2015.

Pirsig, R., Zen and the Art of Motorcycle Maintenance, Londres, Corgi Books, 1976 (ed. orig. 1974).

Quintanilla, M.A., "Engaging Technologies: Criteria for an Alternative Model of Technological Development", en: B. Laspra y J.A. López Cerezo (eds.), Spanish Philosophy of Technology, Dordrecht, Springer, 2018. 
Quintanilla, M.A., M. Parselis, D. Sandrone y D. Lawler, Tecnologías entrañables: ¿es posible un modelo alternativo de desarrollo tecnológico?, Madrid, Libros de la Catarata, 2017.

Renn, O., Risk Governance: Coping with Uncertainty in a Complex World, Londres, Earthscan, 2008.

Rousseau, D.M., S.B. Sitkin, R.S. Burt y C. Camerer, "Not So Different after All: A Cross-Discipline View of Trust", The Academy of Management Review, 22/3 (1998): 393-404.

Sandman, P., Responding to Community Outrage: Strategies for Effective Risk Communication, Falls Curch VA, American Industrial Hygiene Association, 1993.

Siegrist, M., H. Gutsche y C. Keller,'Trust and Confidence in Crisis Communication: Three Case Studies”, en: M. Siegrist, T.C. Earle y H. Gutsche (eds.), Trust in Risk Management: Uncertainty and Scepticism in the Public Mind, Londres-Washington DC, Earthscan, 2007.

Stern, R., “'Trust is Basic': Løgstrup on the Priority of Trust”, en: P. Faulkner y T. Simpson (eds.), The Philosophy of Trust, Oxford, Oxford University Press, 2017.

Sztompka, P., Trust: A Sociological Theory, Cambridge, Cambridge University Press, 2000.

Walaski, P., Risks and Crisis Communication: Methods and Messages, New Jersey, Wiley, 2011.

Warren, M.E., "Trust and Democracy”, en: E. M. Uslaner (ed.), The Oxford Handbook of Social and Political Trust, Nueva York, Oxford University Press, 2018.

Weber, L. y A. Carter, The Social Construction of Trust, Nueva York, Springer, 2003.

Zagzebski, L.T., Virtues of the Mind: An Inquiry into the Nature of Virtue and the Ethical Foundations of Knowledge, Cambridge, Cambridge University Press, 1996. 